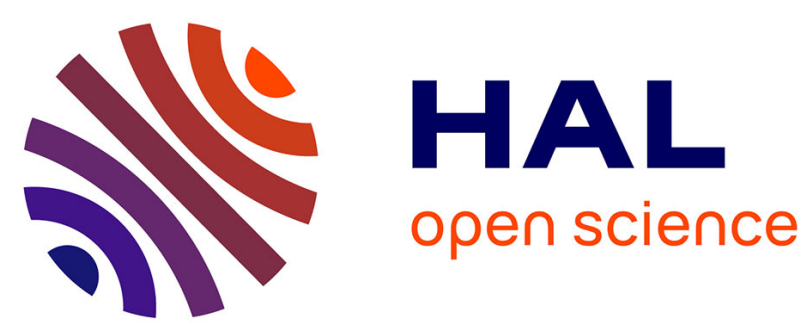

\title{
ENERGY, WATER, AND BROAD-SCALE GEOGRAPHIC PATTERNS OF SPECIES RICHNESS
}

Bradford A Hawkins, Richard Field, Howard V Cornell, David J Currie, Jean-François Guégan, Dawn M Kaufman, Jeremy T Kerr, Gary G Mittelbach, Thierry Oberdorff, Eileen O'Brien, et al.

\section{To cite this version:}

Bradford A Hawkins, Richard Field, Howard V Cornell, David J Currie, Jean-François Guégan, et al.. ENERGY, WATER, AND BROAD-SCALE GEOGRAPHIC PATTERNS OF SPECIES RICHNESS.

Ecology, 2003, 84 (12), pp.3105-3117. 10.1890/03-8006 . hal-02393050

\section{HAL Id: hal-02393050 https://hal.science/hal-02393050}

Submitted on 4 Dec 2019

HAL is a multi-disciplinary open access archive for the deposit and dissemination of scientific research documents, whether they are published or not. The documents may come from teaching and research institutions in France or abroad, or from public or private research centers.
L'archive ouverte pluridisciplinaire HAL, est destinée au dépôt et à la diffusion de documents scientifiques de niveau recherche, publiés ou non, émanant des établissements d'enseignement et de recherche français ou étrangers, des laboratoires publics ou privés. 


\title{
ENERGY, WATER, AND BROAD-SCALE GEOGRAPHIC PATTERNS OF SPECIES RICHNESS
}

\author{
Bradford A. Hawkins, ${ }^{1,12}$ Richard Field, ${ }^{2}$ Howard V. Cornell,${ }^{3}$ David J. Currie, ${ }^{4}$ \\ Jean-François Guégan, ${ }^{5}$ Dawn M. Kaufman, ${ }^{6}$ Jeremy T. Kerr, ${ }^{4}$ Gary G. Mittelbach, ${ }^{7}$ \\ Thierry Oberdorff, ${ }^{8}$ Eileen M. O'Brien, ${ }^{9}$ Eric E. Porter, ${ }^{10}$ And John R. G. Turner ${ }^{11}$ \\ ${ }^{1}$ Department of Ecology and Evolutionary Biology, University of California, Irvine, California 92697 USA \\ ${ }^{2}$ School of Geography, University of Nottingham, University Park, Nottingham NG7 2RD, UK \\ ${ }^{3}$ Department of Biological Sciences, University of Delaware, Newark, Delaware 19716 USA \\ ${ }^{4}$ Department of Biology, University of Ottawa, Ottawa, Ontario, Canada K1N 6N5
}

${ }^{5}$ Centre d'Etude sur le Polymorphisme des Micro-Organismes, CEPM/UMR CNRS-IRD 9926, Equipe: "Evolution des Systemes Symbiotiques," IRD, 911 Avenue Agropolis, B. P. 5045, 34032 Montpellier Cedex 1, France ${ }^{6}$ Division of Biology, Kansas State University, Manhattan, Kansas 66506 USA

${ }^{7}$ Kellogg Biological Station and Department of Zoology, Michigan State University, Hickory Corners, Michigan 49060 USA ${ }^{8}$ Institut de Recherche pour le Développement (IRD), Laboratoire d'Ichtyologie, Muséum National d'Histoire Naturelle, 43, rue Cuvier, 75005 Paris, France

${ }^{9}$ Gainesville College, Gainesville, GA 30503 and Institute of Ecology, University of Georgia, Athens, Georgia 30602 USA ${ }^{10}$ Department of Zoology, Miami University, Oxford, Ohio 45056 USA

${ }^{11}$ Centre for Research in Biodiversity and Conservation, University of Leeds, Leeds LS2 9JT, UK

Abstract. It is often claimed that we do not understand the forces driving the global diversity gradient. However, an extensive literature suggests that contemporary climate constrains terrestrial taxonomic richness over broad geographic extents. Here, we review the empirical literature to examine the nature and form of the relationship between climate and richness. Our goals were to document the support for the climatically based energy hypothesis, and within the constraints imposed by correlative analyses, to evaluate two versions of the hypothesis: the productivity and ambient energy hypotheses. Focusing on studies extending over $800 \mathrm{~km}$, we found that measures of energy, water, or water-energy balance explain spatial variation in richness better than other climatic and non-climatic variables in 82 of 85 cases. Even when considered individually and in isolation, water/ energy variables explain on average over $60 \%$ of the variation in the richness of a wide range of plant and animal groups. Further, water variables usually represent the strongest predictors in the tropics, subtropics, and warm temperate zones, whereas energy variables (for animals) or water-energy variables (for plants) dominate in high latitudes. We conclude that the interaction between water and energy, either directly or indirectly (via plant productivity), provides a strong explanation for globally extensive plant and animal diversity gradients, but for animals there also is a latitudinal shift in the relative importance of ambient energy vs. water moving from the poles to the equator. Although contemporary climate is not the only factor influencing species richness and may not explain the diversity pattern for all taxonomic groups, it is clear that understanding water-energy dynamics is critical to future biodiversity research. Analyses that do not include water-energy variables are missing a key component for explaining broad-scale patterns of diversity.

Key words: diversity gradients; energy hypothesis; latitudinal gradient; productivity hypothesis, species-energy theory; species richness; water-energy dynamics.

\section{INTRODUCTION}

Almost two centuries after the discovery of the "latitudinal gradient" in species richness, a widely ac-

Manuscript received 3 February 2003; revised 17 April 2003; accepted 23 April 2003. Corresponding Editor: J. S. Brewer.

${ }^{12}$ E-mail: bhawkins@uci.edu cepted explanation continues to elude ecologists and biogeographers. A sure indicator of our muddle is that, despite the large number of hypotheses that have been proposed (Pianka 1966, Huston 1979, 1994, Rohde 1992, Rosenzweig 1995), new hypotheses continue to appear (Ritchie and Olff 1999, Colwell and Lees 2000, Dynesius and Jansson 2000), and the total now exceeds 
thirty. But is it true that we lack sufficient evidence to reach a consensus regarding at least the primary factors influencing broad-scale richness gradients, especially at the global scale? We believe that even if we have not conclusively identified the primary mechanism(s) underlying global patterns of richness, it is at least possible to narrow substantially the list of most plausible explanations (see e.g., Rahbek and Graves 2001, Willig et al. 2003).

The energy hypothesis, a climatically based hypothesis that claims that energy availability generates and maintains richness gradients, is a likely candidate for a short list of explanations. The idea that climate's control of energy drives the global richness gradient dates from the beginning of biogeography (von Humboldt 1808) and has generated an extensive literature quantifying the relationship between species richness and climatic variables. Ten years ago, Wright et al. (1993) reviewed this literature and found broadly based support for the energy hypothesis. However, many additional studies of richness gradients have been conducted since then. Moreover, Wright et al. (1993) did not distinguish individual components of climate or consider the possibility that several mechanisms may underlie climate-richness relationships. In this paper, we return to this literature to address four related questions: (1) What is the scope of the empirical evidence that climate represents a primary explanation for richness gradients? (2) How much of the variation in species richness across broad scales can be accounted for by climate? (3) What specific aspects of climate best explain richness? and (4) How might two possible versions of the energy hypothesis contribute to richness gradients? The final question was not addressed by Wright et al. (1993), but is relevant because the energy hypothesis actually subsumes two driving mechanisms (Turner et al. 1987, 1996, Currie 1991, Hawkins et al. 2003).

One version of the hypothesis, as developed by Hutchinson (1959), Connell and Orias (1964), Brown (1981), and Wright (1983), proposes that energy constrains richness via trophic cascades. For example, Wright (1983) argued that at the base of the global food web, plant richness is limited primarily by solar energy and water availability (i.e., water-energy dynamics). Herbivore richness in turn is limited by the net primary production of plants, predator richness is limited by the secondary production of herbivores, and so on up the food chain. In this view, limits on richness are set by the energy flowing through food webs rather than the total energy entering a geographic area. This is often referred to as the productivity hypothesis and has received considerable attention (Wright et al. 1993, Huston 1994, Mittelbach et al. 2001).

The second hypothesis can be traced back to von Humboldt (1808) and is founded on the physiological requirements of organisms vis-à-vis ambient energy inputs rather than food availability. For example, von
Humboldt argued that plant richness declines at higher latitudes because many species are frost-intolerant and cannot survive cold temperate-zone winters. These cold winters are a direct result of seasonal declines in ambient energy as one moves away from the equator. With respect to animals, Turner et al. (1987) argued that sunshine and summer temperatures constrain butterfly richness in Great Britain because adult activity levels depend on ambient temperature and on basking in direct sunlight. Similarly, Currie (1991) hypothesized that thermoregulatory needs explain why the species richness of terrestrial North American vertebrates is more strongly correlated with annual potential evapotranspiration (PET, a measure of ambient or atmospheric energy) than with annual actual evapotranspiration (AET, a measure of water-energy balance closely associated with plant productivity). Whether or not the explanations proposed by von Humboldt (1808), Turner et al. (1987), and Currie (1991) are correct in detail, they represent an alternative mechanism to that represented by the productivity hypothesis. Of course, both physiological and trophic factors may underlie associations between climate and richness, and an additional focus of our analysis is to evaluate where on the globe and for which taxa pure energy variables or water-energy variables best predict diversity gradients.

\section{Methods}

A database of studies of broad-scale richness gradients was compiled by a National Center for Ecological Analysis and Synthesis (NCEAS) working group on species-energy theory. Computerized literature databases, journals, the database generated by Wright et al. (1993), and a productivity-richness database generated by a previous NCEAS working group (Mittelbach et al. 2001) were all searched. A study was included if we could extract coefficients of determination derived from any standard statistical technique for individual explanatory variables for variation in the taxonomic richness (usually species, but occasionally generic or familial) of any terrestrial plant or animal group, although we also included a few studies where pairs of variables were combined by authors. In a few cases, raw data could be extracted from the original paper. If so, we recalculated the models to verify the published results, transforming variables if appropriate and computing coefficients of determination if the original authors had not provided them. Because these data were reanalyzed, the coefficients of determination sometimes differ from those reported in the original publications.

It is clear that the factors influencing richness gradients are scale dependent, and thus, explanations may vary with the spatial scope of the analysis (extent) and with sample resolution (grain) (Willis and Whittaker 2002). Our focus here is on gradients extending over at least $800 \mathrm{~km}$ of linear distance. We considered this distance minimally sufficient to encompass a range of 
climates over which richness may vary. We also examined possible influences of grain size by recording whether the richness data had been generated from range maps (map data) or by sampling flora or fauna (sample data). Maps are usually used for large-grained studies (resolution measured in thousands to hundreds of thousands of square kilometers), whereas finegrained studies (resolution measured in square meters to hectares) use samples of the taxa actually present in plots.

We excluded from consideration papers focused entirely on area, latitude, altitude or "geometric constraints." Clearly, larger areas generally support more species than smaller areas, as long as the climates among the areas are not too different. The importance of climatic consistency among areas becomes obvious if we ask "Which area supports the most plant and animal species, Costa Rica $\left(\sim 51000 \mathrm{~km}^{2}\right)$, the Sahara Desert $\left(\sim 9065000 \mathrm{~km}^{2}\right)$, or Antarctica $(\sim 13340000$ $\left.\mathrm{km}^{2}\right)$ ?" If this comparison seems absurd, it is only because most ecologists accept that climate must be taken into consideration when comparing areas with widely different climatic regimes. As such, analyses that exclude climatic variables when examining species-area relationships across large extents are likely to confound the effects of both variables and cannot be unambiguously interpreted. Second, virtually all workers realize that statistical relationships between richness and latitude or altitude provide no information about underlying driving factors, so studies focusing on these variables were excluded. Finally, we excluded papers focused on geometric constraints on diversity; operationally, because, like studies of latitude or area, correlating diversity with points on the earth's surface without also including other variables makes it impossible to assess the extent that the correlations are due to covariation with spatially patterned environmental factors; more fundamentally, it has been argued that this idea is based on a flawed range concept and cannot explain diversity gradients in either real or null worlds (Hawkins and Diniz-Filho 2002).

\section{RESULTS}

We located 85 data sets: 22 for plants, 44 for vertebrates, and 19 for invertebrates (Table 1). Because of fundamental biological differences among these three groups, we evaluate each separately. However, across all groups, we found that the single best predictor variable explained, on average, $63.4 \%$ of the variance in richness (range 22-98\%). The amount of variance explained does not depend on whether the data are mapvs. sample-based (ANOVA, $F_{1,83}=0.004, P=0.95$, map mean $=63.3 \pm 2.4 \%$, sample mean $=63.6 \pm$ $3.4 \%$ [means $\pm 1 \mathrm{SE}$ ]) or on which group is considered (ANOVA, $F_{2,82}=1.44, P=0.24$, plants mean $=68.9$ $\pm 3.8 \%$, vertebrates mean $=61.2 \pm 2.7 \%$, and invertebrates mean $=62.1 \pm 4.1 \%)$. We also found that climatic variables (whether direct or indirect via plant productivity) were the strongest predictors of richness in 83 of the 85 cases, offering widespread support for the hypothesis that climate in general has a major influence on diversity gradients across large spatial extents. However, there also is clear taxonomically and geographically based heterogeneity in the data with respect to the specific climatic variables most strongly associated with richness, relevant to the question of whether water, energy, or their interaction may underlie diversity gradients.

\section{Plant richness}

In 20 of 21 cases, variables including water inputs emerge as the primary predictor of plant richness (Table 1 ), based on the $r^{2}$ values of each tested variable. The specific variables found reflect the variables included in each study, but all represent measures of water (rainfall and precipitation) or water-energy (actual evapotranspiration, productivity, net primary productivity, and net aboveground productivity). Further, water variables tend to be the best predictors when the geographic scope of the data is restricted to tropical and subtropical areas, whereas water-energy variables dominate when colder areas are also included. The single exception to the general result is for North American trees, where a reanalysis of Currie and Paquin's (1987) data found that a temperature index explained two/thirds of the variance (Table 1; Allen et al. 2002). However, the analysis of Allen et al. (2002) was specifically designed to support a temperature-based hypothesis and did not include any water-related variables. Clearly, the data strongly implicate water availability as a key constraint on plant richness, particularly in warm areas where energy is abundant. In cold regions where energy inputs are lower and thus more likely to be limiting, energy interacts with water to explain richness gradients. It is notable that energy inputs alone never represent the primary explanatory variable for plant richness gradients, unless both water and water-energy variables are excluded from the analysis. Thus, a parsimonious interpretation of the plant studies is that plant richness is determined largely by water-energy dynamics, although pure water variables may be most strongly associated with diversity in warm areas.

\section{Vertebrate richness}

The relationships between water, energy, and richness show a wider range of variation for vertebrates than for plants (Table 1). In 24 cases, water or waterenergy (measured either directly or indirectly as plant productivity) explained the most variance in richness, whereas in 17 cases, direct energy measures dominate. However, there are taxonomic and geographical patterns in the results. First, seven of the latter cases involve reptiles/lizards, suggesting that the richness of at least some reptile groups is most strongly constrained by ambient energy inputs, wherever they occur.

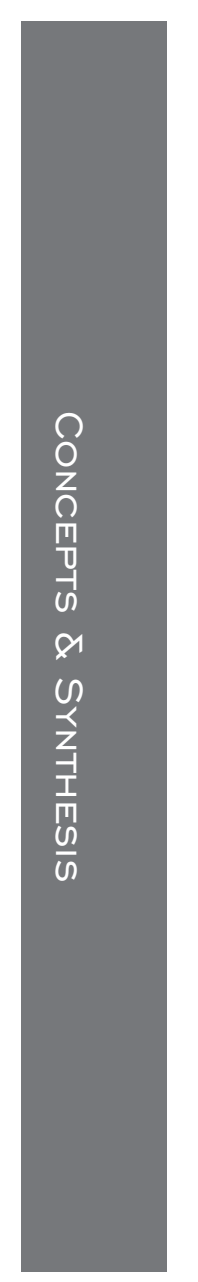


TABLE 1. Primary explanatory variables in analyses of broad-scale species richness patterns (i.e., extent $>800 \mathrm{~km}$ ) of plants, vertebrates, and invertebrates; other classes of variables included in each study are also listed.

\begin{tabular}{|c|c|c|c|c|c|c|}
\hline $\begin{array}{l}\text { Taxonomic } \\
\text { group }\end{array}$ & $\begin{array}{l}\text { Geographic } \\
\text { area }\end{array}$ & $\begin{array}{l}\text { Data } \\
\text { type } \dagger\end{array}$ & $\begin{array}{c}\text { Primary } \\
\text { variable }\end{array}$ & $\begin{array}{c}\text { Other } \\
\text { variables§ }\end{array}$ & $r^{2}$ & Reference \\
\hline \multicolumn{7}{|l|}{ Plants } \\
\hline Plants & global & samples & productivity & E,W,WE,T,A,O & 0.28 & $\begin{array}{l}\text { Scheiner and Rey- } \\
\text { Benayas (1994) }\end{array}$ \\
\hline Plants & global (islands) & maps & AET & A & 0.76 & Wright (1983) \\
\hline Plants & California & maps & precipitation & $\mathrm{E}, \mathrm{T}, \mathrm{A}, \mathrm{O}$ & 0.52 & $\begin{array}{l}\text { Richerson and } \\
\text { Lum (1980) }\end{array}$ \\
\hline $\begin{array}{l}\text { Angiosperm } \\
\text { families }\end{array}$ & global & maps & $\mathrm{PET}+\mathrm{WD}$ & $\mathrm{O}$ & 0.84 & $\begin{array}{l}\text { Francis and Currie } \\
(2003)\end{array}$ \\
\hline Woody plants & global & samples & rainfall & $\mathrm{T}, \mathrm{O}$ & 0.74 & Gentry (1988) \\
\hline Woody plants & N. Neotropics & samples & rainfall & $\ldots$ & 0.86 & Gentry (1982) \\
\hline Woody plants & N. Neotropics & samples & rainfall & $\mathrm{T}, \mathrm{O}$ & 0.53 & $\begin{array}{l}\text { Clinebell et al. } \\
\text { (1995) }\end{array}$ \\
\hline Woody plants & S. Africa & maps & rainfall & E,WE,T,O & 0.60 & O’Brien (1993) \\
\hline Woody plants & Australia & samples & productivity & ... & 0.98 & $\begin{array}{l}\text { Specht and Specht } \\
\text { (1994) }\end{array}$ \\
\hline Trees & N. temperate zone & samples & AET & $\mathrm{A}, \mathrm{O}$ & 0.67 & $\begin{array}{l}\text { Francis and Currie } \\
\text { (1998) }\end{array}$ \\
\hline Trees & N. America & maps & AET & E,W,T,O & 0.62 & $\begin{array}{l}\text { Currie and Paquin } \\
\text { (1987) }\end{array}$ \\
\hline Trees & N. America & maps & NPP (modeled) & E,T,O & 0.91 & $\begin{array}{l}\text { Adams and Wood- } \\
\text { ward (1989) }\end{array}$ \\
\hline Trees & Europe & maps & NPP (modeled) & $\mathrm{E}, \mathrm{T}, \mathrm{O}$ & 0.84 & $\begin{array}{l}\text { Adams and Wood- } \\
\text { ward (1989) }\end{array}$ \\
\hline Trees & E. Asia & maps & NPP (modeled) & $\mathrm{E}, \mathrm{T}, \mathrm{O}$ & 0.98 & $\begin{array}{c}\text { Adams and Wood- } \\
\text { ward (1989) }\end{array}$ \\
\hline Trees & S. America & samples & rainfall & $\ldots$ & 0.65 & Kay et al. (1997) \\
\hline Trees & Madagascar & samples & rainfall & $\cdots$ & 0.50 & $\begin{array}{l}\text { Ganzhorn et al. } \\
\text { (1997) }\end{array}$ \\
\hline Trees & N. America & maps & temperature & 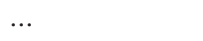 & 0.66 & Allen et al. (2002) \\
\hline Bog plants & $\begin{array}{c}\text { northeastern } \mathrm{N} . \\
\text { America }\end{array}$ & samples & rainfall & $\mathrm{E}, \mathrm{A}, \mathrm{O}$ & 0.60 & Glaser (1992) \\
\hline Herbs/shrubs & Chile & samples & rainfall & $\ldots$ & 0.70 & $\begin{array}{l}\text { Meserve and } \\
\text { Glanz (1978) }\end{array}$ \\
\hline Pteridophytes & Bolivia & samples & precipitation & E,T,A,O & 0.64 & Kessler (2001) \\
\hline Cacti & Argentina & maps & summer rainfall & E,WE,O & 0.50 & $\begin{array}{l}\text { Mourelle and } \\
\text { Ezcurra (1996) }\end{array}$ \\
\hline Grasses & Namibia & samples & rainfall & $\ldots$ & 0.77 & $\begin{array}{l}\text { Schulze et al. } \\
\quad(1996)\end{array}$ \\
\hline \multicolumn{7}{|l|}{ Vertebrates } \\
\hline Vertebrates & Afrotropics & maps & NPP (modeled) & $\mathrm{T}, \mathrm{O}$ & 0.73 & $\begin{array}{l}\text { Balmford et al. } \\
(2001)\end{array}$ \\
\hline Vertebrates & N. America & maps & PET & W,WE,T,O & 0.92 & Currie (1991) \\
\hline Birds & Global (islands) & maps & NPP (modeled) & A & 0.80 & Wright (1983) \\
\hline Birds & $\begin{array}{l}\text { Global (conti- } \\
\text { nents) }\end{array}$ & maps & AET & $\mathrm{E}, \mathrm{W}, \mathrm{T}, \mathrm{O}$ & 0.72 & $\begin{array}{l}\text { Hawkins et al. } \\
(2003)\end{array}$ \\
\hline Birds & India & maps & rainfall & $\cdots$ & 0.51 & $\begin{array}{l}\text { Pearson and Car- } \\
\text { roll (1998) }\end{array}$ \\
\hline Birds & S. Africa & maps & precipitation & E,WE,O & 0.67 & $\begin{array}{l}\text { van Rensburg et } \\
\text { al. }(2002)\end{array}$ \\
\hline Birds & Australia & maps & rainfall & $\mathrm{E}, \mathrm{O}$ & 0.48 & $\begin{array}{l}\text { Pianka and Schall } \\
\text { (1981) }\end{array}$ \\
\hline Birds & $\begin{array}{l}\text { USA }+ \text { southern } \\
\text { Canada }\end{array}$ & samples & NDVI & $\mathrm{T}$ & 0.61 & $\begin{array}{l}\text { Hurlbert and Has- } \\
\text { kell (2003) }\end{array}$ \\
\hline Birds & USSR & maps & temperature & $\mathrm{W}$ & 0.89 & Terent'ev (1963) \\
\hline Birds & Norway & samples & May temperature & $\cdots$ & 0.78 & $\begin{array}{l}\text { Heggberget } \\
\text { (1987) }\end{array}$ \\
\hline $\begin{array}{l}\text { Birds } \\
\quad \text { (summer) }\end{array}$ & UK & maps & $\begin{array}{l}\text { summer tempera- } \\
\text { ture }\end{array}$ & $\mathrm{W}, \mathrm{T}, \mathrm{O}$ & 0.34 & $\begin{array}{l}\text { Lennon et al. } \\
\text { (2000) }\end{array}$ \\
\hline Birds (winter) & UK & maps & $\begin{array}{l}\text { summer tempera- } \\
\text { ture }\end{array}$ & $\mathrm{W}, \mathrm{T}, \mathrm{O}$ & 0.59 & $\begin{array}{l}\text { Lennon et al. } \\
\text { (2000) }\end{array}$ \\
\hline $\begin{array}{l}\text { Passerine } \\
\text { birds }\end{array}$ & Argentina & maps & temperature & $\mathrm{W}, \mathrm{T}, \mathrm{O}$ & 0.57 & $\begin{array}{l}\text { Rabinovich and } \\
\text { Rapoport } \\
(1975)\end{array}$ \\
\hline Amphibians & Europe & maps & AET & E,W,P,T,O & 0.61 & $\begin{array}{l}\text { M. A. Rodriguez, } \\
\text { J. A. Belmon- } \\
\text { tes, and B. A. } \\
\text { Hawkins (un- } \\
\text { published data) }\end{array}$ \\
\hline
\end{tabular}


TABle 1. Continued.

\begin{tabular}{|c|c|c|c|c|c|c|}
\hline $\begin{array}{l}\text { Taxonomic } \\
\text { group }\end{array}$ & $\begin{array}{l}\text { Geographic } \\
\text { area }\end{array}$ & $\begin{array}{l}\text { Data } \\
\text { type } †\end{array}$ & $\begin{array}{c}\text { Primary } \\
\text { variable } \ddagger\end{array}$ & $\begin{array}{c}\text { Other } \\
\text { variables§ }\end{array}$ & $r^{2}$ & Reference \\
\hline Amphibians & Iberia & maps & precipitation & $\mathrm{E}, \mathrm{O}$ & 0.22 & $\begin{array}{l}\text { Schall and Pianka } \\
\text { (1977) }\end{array}$ \\
\hline Amphibians & N. America & maps & temperature & $\ldots$ & 0.69 & Allen et al. (2002) \\
\hline Amphibians & USSR & maps & temperature & W & 0.46 & Terent'ev (1963) \\
\hline Frogs & USA & maps & rainfall & $\mathrm{E}, \mathrm{T}, \mathrm{O}$ & 0.64 & $\begin{array}{l}\text { Schall and Pianka } \\
\text { (1978) }\end{array}$ \\
\hline Frogs & Australia & maps & rainfall & $\mathrm{E}, \mathrm{O}$ & 0.53 & $\begin{array}{l}\text { Pianka and Schall } \\
\text { (1981) }\end{array}$ \\
\hline Mammals & N. Neotropics & samples & rainfall & $\mathrm{T}, \mathrm{A}, \mathrm{O}$ & 0.48 & Medellin (1994) \\
\hline Mammals & Chile & samples & precipitation & $\mathrm{O}$ & 0.90 & $\begin{array}{l}\text { Meserve and } \\
\text { Glanz (1978) }\end{array}$ \\
\hline Mammals & USSR & maps & temperature & W & 0.89 & Terent'ev (1963) \\
\hline Mammals & N. America & maps & temperature & W,WE,T & 0.57 & $\begin{array}{l}\text { Badgley and Fox } \\
(2000)\end{array}$ \\
\hline Mammals & S. Africa & maps & $\begin{array}{l}\text { variability in } \\
\text { temperature }\end{array}$ & W,WE,T,O & 0.69 & $\begin{array}{l}\text { Andrews and } \\
\text { O'Brien (2000) }\end{array}$ \\
\hline Mammals & N. Palearctic & maps & $\begin{array}{l}\text { percentage of } \\
\text { forest }\end{array}$ & $\mathrm{E}, \mathrm{O}$ & 0.79 & $\begin{array}{l}\text { Danell et al. } \\
\quad(1996)\end{array}$ \\
\hline Mammals & N. Nearctic & maps & $\begin{array}{l}\text { forest fragmenta- } \\
\quad \text { tion }\end{array}$ & $\mathrm{E}, \mathrm{O}$ & 0.45 & $\begin{array}{l}\text { Danell et al. } \\
\text { (1996) }\end{array}$ \\
\hline Marsupials & Australia & maps & rainfall & $\mathrm{E}, \mathrm{O}$ & 0.54 & $\begin{array}{l}\text { Pianka and Schall } \\
\text { (1981) }\end{array}$ \\
\hline Primates & S. America & samples & rainfall & $\mathrm{P}, \mathrm{O}$ & 0.37 & Kay et al. (1997) \\
\hline Primates & S. America & samples & rainfall & $\cdots$ & 0.67 & $\begin{array}{l}\text { Reed and Fleagle } \\
\text { (1995) }\end{array}$ \\
\hline Primates & Africa & samples & rainfall & $\cdots$ & 0.75 & $\begin{array}{l}\text { Reed and Fleagle } \\
\text { (1995) }\end{array}$ \\
\hline Primates & Madagascar & samples & rainfall & $\cdots$ & 0.70 & $\begin{array}{l}\text { Reed and Fleagle } \\
\quad(1995)\end{array}$ \\
\hline Lemurs & Madagascar & samples & rainfall & $\mathrm{O}$ & 0.46 & $\begin{array}{l}\text { Ganzhorn et al. } \\
\text { (1997) }\end{array}$ \\
\hline Rodents & southwestern USA & samples & precipitation & $\mathrm{O}$ & 0.44 & Brown (1973) \\
\hline Reptiles & Europe & maps & PET & W,WE,P,T,O & 0.71 & $\begin{array}{l}\text { M. A. Rodriguez, } \\
\text { J. A. Belmon- } \\
\text { tes, and B. A. } \\
\text { Hawkins (un- } \\
\text { published data) }\end{array}$ \\
\hline Reptiles & USSR & maps & temperature & W & 0.90 & Terent'ev (1963) \\
\hline Reptiles & Iberia & maps & hours of sunshine & $\mathrm{W}, \mathrm{O}$ & 0.36 & $\begin{array}{l}\text { Schall and Pianka } \\
\text { (1977) }\end{array}$ \\
\hline Snakes & Australia & maps & rainfall & $\mathrm{E}, \mathrm{O}$ & 0.57 & $\begin{array}{l}\text { Pianka and Schall } \\
\text { (1981) }\end{array}$ \\
\hline Snakes & USA & maps & rainfall & $\mathrm{E}, \mathrm{T}, \mathrm{O}$ & 0.32 & $\begin{array}{l}\text { Schall and Pianka } \\
\text { (1978) }\end{array}$ \\
\hline Turtles & Australia & maps & rainfall & $\mathrm{E}, \mathrm{O}$ & 0.77 & $\begin{array}{l}\text { Pianka and Schall } \\
\text { (1981) }\end{array}$ \\
\hline Turtles & USA & maps & rainfall & $\mathrm{E}, \mathrm{T}, \mathrm{O}$ & 0.53 & $\begin{array}{l}\text { Schall and Pianka } \\
\text { (1978) }\end{array}$ \\
\hline Lizards & USA & maps & hours of sunshine & $\mathrm{W}, \mathrm{T}, \mathrm{O}$ & 0.67 & $\begin{array}{l}\text { Schall and Pianka } \\
\text { (1978) }\end{array}$ \\
\hline Lizards & $\begin{array}{l}\text { western N. Amer- } \\
\text { ica }\end{array}$ & samples & July temperature & $\mathrm{W}, \mathrm{T}, \mathrm{O}$ & 0.88 & Pianka (1967) \\
\hline Lizards & western USA & samples & Jan temperature & $\mathrm{W}, \mathrm{T}, \mathrm{O}$ & 0.32 & Scheibe (1987) \\
\hline Lizards & Australia & maps & temperature & $\mathrm{W}, \mathrm{O}$ & 0.44 & $\begin{array}{l}\text { Pianka and Schall } \\
\text { (1981) }\end{array}$ \\
\hline \multicolumn{7}{|l|}{ Invertebrates } \\
\hline Tiger beetles & N. America & maps & PET & W,WE,O & 0.87 & $\begin{array}{l}\text { Kerr and Currie } \\
\text { (1999) }\end{array}$ \\
\hline Tiger beetles & India & maps & rainfall & $\cdots$ & 0.58 & $\begin{array}{l}\text { Pearson and Car- } \\
\text { roll (1998) }\end{array}$ \\
\hline Tiger beetles & Australia & maps & rainfall & $\cdots$ & 0.25 & $\begin{array}{l}\text { Pearson and Car- } \\
\text { roll (1998) }\end{array}$ \\
\hline $\begin{array}{l}\text { Epicauta } \\
\text { (beetles) }\end{array}$ & N. America & maps & PET & W,WE,T,O & 0.82 & $\begin{array}{l}\text { Kerr and Packer } \\
\text { (1999) }\end{array}$ \\
\hline Dung beetles & France & maps & temperature & $\mathrm{W}, \mathrm{T}, \mathrm{O}$ & 0.36 & Lobo et al. (2002) \\
\hline Butterflies & W. Palearctic & maps & $\mathrm{AET}$ & $\mathrm{E}, \mathrm{W}, \mathrm{T}, \mathrm{O}$ & 0.79 & $\begin{array}{l}\text { B. A. Hawkins } \\
\text { and E. E. Porter } \\
\text { (unpublished } \\
\text { data) }\end{array}$ \\
\hline
\end{tabular}


TABle 1. Continued.

\begin{tabular}{|c|c|c|c|c|c|c|}
\hline $\begin{array}{l}\text { Taxonomic } \\
\text { group }\end{array}$ & $\begin{array}{l}\text { Geographic } \\
\text { area }\end{array}$ & $\begin{array}{l}\text { Data } \\
\text { type } \dagger\end{array}$ & $\begin{array}{c}\text { Primary } \\
\text { variable } \neq\end{array}$ & $\begin{array}{c}\text { Other } \\
\text { variables§ }\end{array}$ & $r^{2}$ & Reference \\
\hline Butterflies & Afrotropics & maps & AET & $\mathrm{E}, \mathrm{W}, \mathrm{T}, \mathrm{O}$ & 0.69 & $\begin{array}{l}\text { B. A. Hawkins } \\
\text { and E. E. Porter } \\
\text { (unpublished } \\
\text { data) }\end{array}$ \\
\hline Butterflies & Australia & maps & AET & $\mathrm{E}, \mathrm{W}, \mathrm{T}, \mathrm{O}$ & 0.62 & $\begin{array}{l}\text { B. A. Hawkins } \\
\text { and E. E. Porter } \\
\text { (unpublished } \\
\text { data) }\end{array}$ \\
\hline Butterflies & Australia & maps & summer rainfall & $\mathrm{E}, \mathrm{T}, \mathrm{O}$ & 0.64 & $\begin{array}{l}\text { Dingle et al. } \\
(2000)\end{array}$ \\
\hline Butterflies & UK & maps & $\begin{array}{l}\text { summer sunshine } \\
\quad+\text { summer } \\
\text { temperature }\end{array}$ & $\cdots$ & 0.79 & $\begin{array}{l}\text { Turner et al. } \\
\text { (1987) }\end{array}$ \\
\hline Butterflies & N. America & maps & PET & W,WE,T,O & 0.69 & $\begin{array}{l}\text { B. A. Hawkins } \\
\text { and E. E. Porter } \\
\text { (unpublished } \\
\text { data) }\end{array}$ \\
\hline Butterflies & Canada & samples & $\begin{array}{l}\text { landcover diver- } \\
\text { sity }\end{array}$ & $\mathrm{E}, \mathrm{W}, \mathrm{WE}, \mathrm{T}$ & 0.74 & Kerr et al. (2001) \\
\hline Moths & UK & maps & $\begin{array}{l}\text { summer sunshine } \\
+ \text { temperature }\end{array}$ & $\ldots$ & 0.72 & $\begin{array}{l}\text { Turner et al. } \\
\text { (1987) }\end{array}$ \\
\hline $\begin{array}{l}\text { Forest Lepi- } \\
\text { doptera }\end{array}$ & Canada & maps & PET & WE,T,O & 0.69 & Kerr et al. (1998) \\
\hline Termites & Africa & maps & NPP & $\cdots$ & 0.39 & $\underset{(1994)}{\text { Eggleton }}$ et al. \\
\hline Termites & Neotropics & maps & NPP & $\cdots$ & 0.41 & $\underset{(1994)}{\text { Eggleton }}$ et al. \\
\hline Termites & Indo-Malaysia & maps & NPP & $\cdots$ & 0.46 & $\underset{(1994)}{\text { Eggleton et al. }}$ \\
\hline Ants & W. Hemisphere & maps & NAP (modeled) & $\cdots$ & 0.58 & $\begin{array}{l}\text { Kaspari et al. } \\
\text { (2000) }\end{array}$ \\
\hline Ants & southwestern USA & samples & rainfall & $\mathrm{E}, \mathrm{O}$ & 0.70 & Davidson (1977) \\
\hline
\end{tabular}

Note: The coefficients of determination $\left(r^{2}\right)$ refer to the explanatory power of the primary variable only.

$\dagger$ Maps $=$ richness generated using range maps, and samples $=$ richness estimated from samples.

\$ Abbreviations are: AET = actual evapotranspiration; $\mathrm{WD}=$ water deficit; $\mathrm{PET}=$ potential evapotranspiration; $\mathrm{NPP}=$ net primary productivity; NDVI = normalized difference vegetation index; NAP $=$ net aboveground productivity.

$\S$ Abbreviations are: $\mathrm{E}=$ energy, $\mathrm{W}=$ water, $\mathrm{WE}=$ water-energy, $\mathrm{P}=$ productivity; $\mathrm{T}=$ topography, $\mathrm{A}=$ area, $\mathrm{O}=$ other, and ellipses $(\cdots)=$ none.

Second, eight of the energy cases not involving reptiles represent studies encompassing regions with widespread cold climates (North America, Norway, Great Britain, and the former Soviet Union). This is in contrast to studies identifying either water as the best predictor (in regions dominated by warm climates) or water-energy variables (in globally extensive data and one case in Europe). However, there are four apparent exceptions. The first involves passerine birds in Argentina (Rabinovich and Rapoport 1975). A major portion of Argentina is mild temperate, yet temperature emerged as the best predictor of bird richness. On the other hand, southern Argentina does include sub-Antarctic climatic areas, and it is likely that temperature is limiting in the far southern hemisphere, as it is in the far northern hemisphere. Another exception was found for the mammals of southern Africa, but in this case, the best climatic predictor was neither energy nor water, but a measure of seasonality (Andrews and O'Brien 2000). This represents the only case we found that identified seasonal variability as predicting richness patterns better than annual climatic variables, and as such, represents an exception to the more general patterns. A study of herbivorous mammals in the northern boreal zone (Danell et al. 1996) also appears to be exceptional, as measures of forest size were the best predictors of richness in both the Palearctic and Nearctic (Table 1). However, based on their full analysis, they concluded that mammal richness was probably temperature limited, consistent with other studies of mammals in the northern temperate zone (Table 1). Thus, despite some possible exceptions, the data for most vertebrates indicate that water-energy variables best account for globalscale patterns, whereas in warm climates, water or water-energy is most strongly associated with richness, and in cold climates ambient energy inputs become critical.

\section{Invertebrate richness}

Although there are fewer data, invertebrates show a pattern very similar to that for vertebrates (Table 1). Water-energy or water variables are the primary predictors, except in some studies encompassing colder regions in the northern temperate zone (North America, Great Britain, and Canada). The case of dung beetles in France might represent an exception, in that they are 


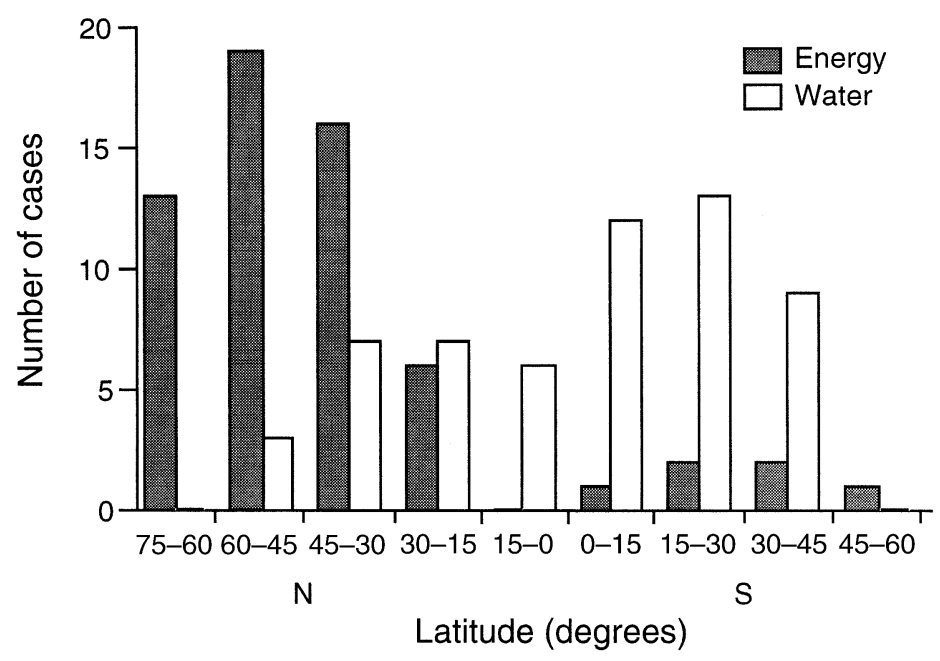

farther south and temperature is still the primary predictor (Lobo et al. 2002), but we will return to this case later. A study of Canadian butterflies (Kerr et al. 2001) represents a case in which climate was not the best predictor, although it could be argued that landcover diversity is itself under climatic control. Nevertheless, the geographic distribution of studies of invertebrates is, overall, consistent with what has been found in vertebrates; energy has a strong relationship with richness in cool climates, whereas water by itself or in conjunction with energy is critical in warmer climates.

\section{Latitude and constraints on diversity}

As indicated in the previous two sections, studies focused on vertebrates or invertebrates suggest a shift in the primary limiting factor from energy to water as one moves from the poles to the equator. To explore this further, we selected the 38 cases for which animal richness patterns were best described by pure energy (PET or temperature) or pure water (rainfall or precipitation), which allowed us to isolate the separate components of water-energy. We then divided the world into nine latitudinal bands of $15^{\circ}$ each, and scored which bands each study encompassed. We then summed all of the studies within each band with respect to the best explanatory variable and plotted the totals against latitude. Although we cannot analyze the resulting pattern statistically because most studies contributed data to multiple bands, the pattern (Fig. 1) reinforces the conclusion that the primary constraint on animal richness varies latitudinally. In high latitudes of the northern hemisphere, energy is most often the primary explanatory variable, with increasing numbers of cases in which water is the strongest predictor as we move south. In the southern hemisphere, water is by far the dominant explanatory variable, except in cases involving reptiles and Argentinean birds. It also appears that the latitudinal transition in the northern hemisphere from energy to water as the primary predictor is gradual, extending over $45^{\circ}$ of latitude, and there a strong asymmetry between the northern and southern hemispheres as a whole (Fig. 1). We will return to these issues in the Discussion.

\section{DISCUSSION}

Our synthesis extends the earlier work of Wright et al. (1993) and provides basic answers to the questions raised in the Introduction. Climate is typically a strong descriptor of broad-scale richness patterns, and the single best climatic predictor on average explains over $60 \%$ of the variance. Also, both water and energy inputs play a prominent role in richness patterns of a wide range of taxonomic groups comprising both plants and animals, supporting the hypothesis that "water-energy dynamics" provides a potent explanation for the diversity gradient across the entire planet (see e.g., O'Brien 1998). Finally, the geographic pattern found among the studies also suggests that the relative importance of the two components of water-energy dynamics shifts latitudinally, such that energy places strong constraints on plant and animal richness in the far north (and probably the far south) where energy inputs are low, whereas water constrains richness in areas with high energy inputs. Thus, a major conclusion is that, if water drives plant production in warm climates as is widely assumed, both the ambient energy and productivity versions of the energy hypothesis find support, depending where in the world the study is focused and on overall water-energy inputs. However, although the data indicate a shift in the primary constraint on richness, the semi-quantitative method we used to compare across latitudes (Fig. 1) is not clear about where on the planet this shift may occur, or if the transition is gradual or abrupt. This is particularly true since curvilinear relationships between energy variables and richness are commonly found in analyses focused on northern latitudes. To examine this in more 
a) Palearctic birds

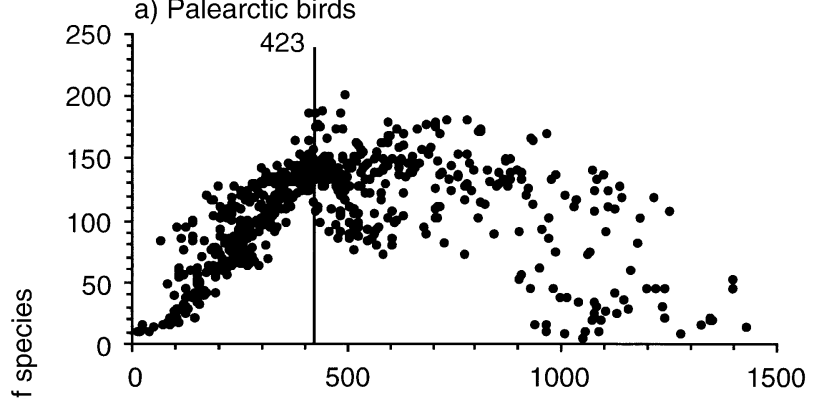

c) North American birds

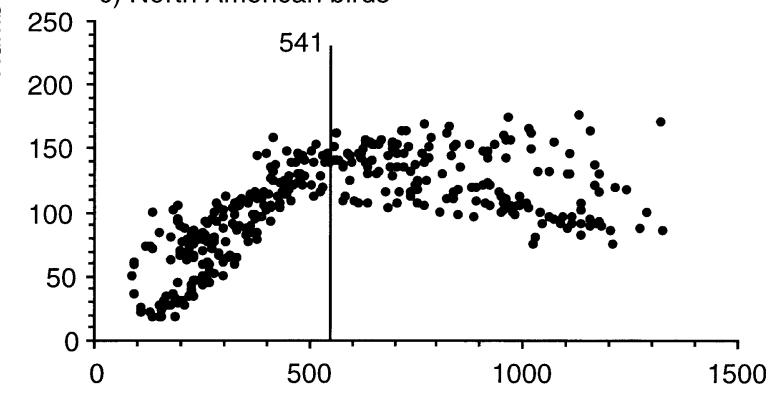

b) Western Palearctic butterflies

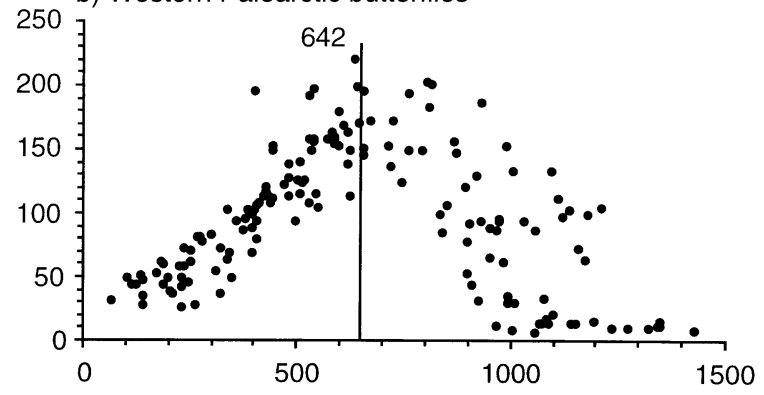

d) North American butterflies

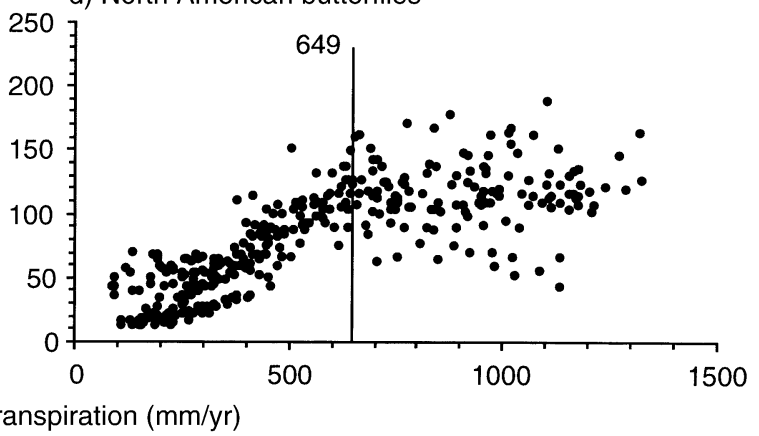

Potential evapotranspiration ( $\mathrm{mm} / \mathrm{yr})$

FIG. 2. Relationships between potential evapotranspiration (PET) and species richness for terrestrial birds (a, c) and butterflies (b, d) in the Palearctic (a, b) and Nearctic (c, d). The vertical lines represent breakpoints identified by split-line regression at which the relationships shift from being positive to either negative (in the Palearctic) or null (in the Nearctic). The regressions were performed using JMP 4.0 (SAS Institute 2000).

detail, we followed Kerr and Packer (1997) in using split-line regression to determine the nature of the relationship between energy and animal richness gradients in the northern hemisphere, where cold terrestrial climates are most widespread and, hence, where energy inputs are often low.

Currie's (1991) finding that vertebrate richness in North America was best described by PET was based on a curvilinear regression model. Kerr and Packer (1997) reanalyzed the mammal data and confirmed that richness was strongly positively associated with potential evapotranspiration above $45^{\circ}-48^{\circ} \mathrm{N}$ (near the USA-Canadian border), but not below this latitude. They proposed this boundary as the threshold below which ambient energy no longer limits mammal richness. To test the generality of this result, we examined the relationship between species richness and potential evapotranspiration using the terrestrial birds of North America and the Palearctic (Hawkins et al. 2003) and the butterflies of North America and the western Palearctic (B. A. Hawkins and E. E. Porter, unpublished data). Richness of both taxa was estimated in equalarea grid cells of $48400 \mathrm{~km}^{2}$ using range maps, and PET was estimated from a global database (available online). ${ }^{13}$

Consistent with other studies in the northern temperate zone (e.g., Currie 1991), both butterfly and bird

${ }^{13}$ URL: 〈http://www.grid.unep.ch/data/grid/gnv183.html〉 richness are strongly correlated with PET at low energy levels, but show either no relationship (Nearctic) or a negative relationship (Palearctic) at higher energy levels (Fig. 2). Split-line regressions further indicate that bird richness is primarily energy-limited only in Canada, the British Isles, Scandinavia, and across northern European Russia and Siberia (Fig. 3). Butterflies appear to be more sensitive to low energy than birds, showing strong positive relationships with PET across Canada and the extreme northern USA, and in most of Europe (Fig. 3). Although the abruptness of the shift from strong to weak associations of PET and richness is arguable, south of these lines it is probable that water replaces energy as the primary constraint (Table 1, Fig. 1 ). Whether these energy thresholds apply to other vertebrate and invertebrate groups remains to be determined, although the threshold for North American mammals (Kerr and Packer 1997) is very similar to that of North American butterflies, and French dung beetle richness is most strongly explained by temperature (Table 1), as would be predicted from the threshold found for European butterflies (Fig. 2). But based on the available evidence, it appears that energy is a strong predictor of animal diversity gradients in only a small part of the planet, and that over most of the earth the distribution of rainfall has a stronger influence on diversity gradients than temperature.

A final point about the geographic distribution of energy and water as predictors of diversity is that we 


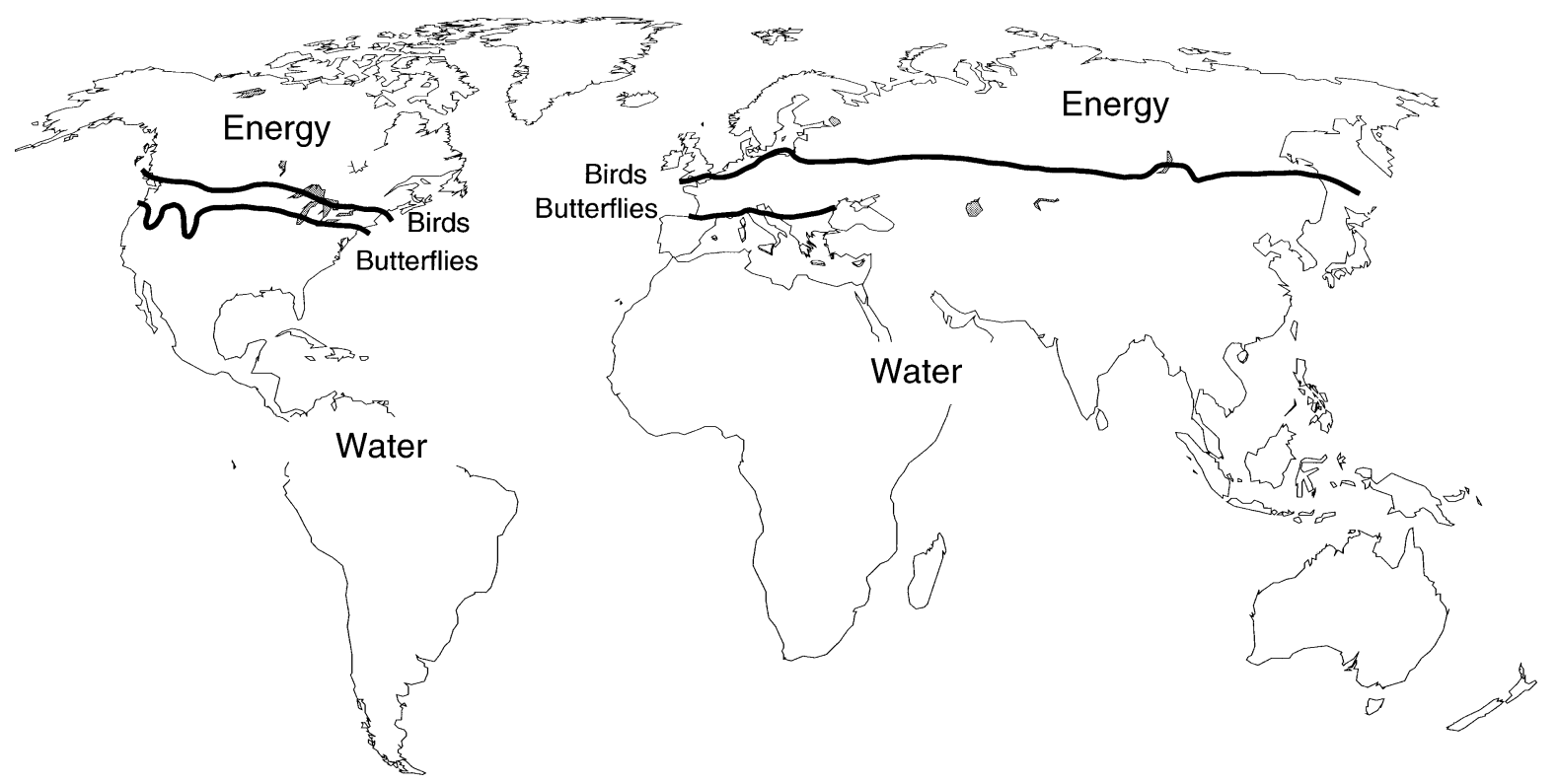

FIG. 3. A hypothesis for the geographic distribution of the limits to the species richness of animals, based on the analysis of butterflies and birds (see Fig. 2). The bold lines represent the geographical distribution of the breakpoints above which potential evapotranspiration and species richness are not positively associated. Across all latitudes, animal richness is constrained by the interaction of energy and water, but north of these lines energy is hypothesized to represent the limiting component of the interaction, whereas south of these lines water is assumed to be the key limiting component (see Fig. 1).

might expect the pattern to be roughly symmetrical around the equator, but it is not (Fig. 1). We consider it likely that the observed asymmetry reflects the fact that interior continental climates are primarily restricted to the northern hemisphere. In the southern hemisphere, climates in the temperate zone are generally much warmer, and even in the far south temperature fluctuations are more buffered by the oceans (outside of Antarctica there is no southern equivalent to Siberian winters), whereas precipitation can still be highly variable because of orographic effects. Thus, although low temperatures are likely to be critical in some parts of the southern hemisphere, such as southern Argentina and at high altitudes, the data suggest that water is more important across very broad geographic areas.

Reptiles, particularly lizards, are commonly found to be most closely associated with energy measures wherever they are studied. Because they are extreme solar ectotherms with a complex array of physiological and behavioral mechanisms for maintaining their body temperatures, it is believed that ambient temperature represents the most important climatic factor influencing reptiles (Heatwole 1976), a view supported by richness studies. The failure of the other major ectothermic vertebrate group, the amphibians, to show the same dependence on temperature at all levels is not surprising, given that most species require moisture to reproduce. Invertebrates are more puzzling. One potential explanation for why they do not follow the pattern found for reptiles is that their small body sizes create problems with desiccation in hot environments. Alter- natively, both water and energy are critical to plants, and invertebrate richness may be linked to plant production, so the influence of water in warm environments is primarily indirect, operating via effects on food availability.

Whether energy and/or water influences both invertebrate and vertebrate richness gradients via direct physiological effects or via plant productivity is key to understanding the mechanism(s) underlying climatically driven richness gradients. However, in most cases, the alternative hypotheses are difficult to disentangle, particularly since they are not mutually exclusive. Plant productivity is closely associated with water-energy balance at the global scale (e.g., Rosenzweig 1968, Lieth 1975), and although new remote-sensing techniques now provide alternative measurements of net primary productivity (Goetz et al. 2000), in the past it has usually been modeled with climatic variables rather than measured directly (see also Huston 1999). Given these problems, it is difficult to decouple climatic vs. productivity influences on richness using multiple regression, the most common statistical technique. Consequently, care is necessary when attempting to determine whether it is more appropriate to refer to observed climate-richness links as supporting a physiologically based "water-energy dynamics" hypothesis or a trophically based "productivity" hypothesis, at least for animals. This complication does not apply to plants, as it is widely accepted that both water and energy are essential to plant physiological processes and thus have direct effects on plant abundance and diversity (Ste- 
phenson 1990, O'Brien 1993). Given the broadly based evidence for the influence of water and energy on animal as well as plant richness, an important next step will be to determine the relative importance of direct and indirect climatic effects on geographic patterns of animals. The finding that pure energy variables usually describe animal diversity gradients best in the far northern latitudes suggests that direct physiological effects dominate in cold climates, but additional work is needed to confirm this interpretation.

A closely related issue is that a set of correlations among variables does not in itself show that they are linked by any causal mechanism, and there have been difficulties imagining mechanisms linking energy or water-energy to diversity gradients, especially if the theory is restricted to deterministic modeling (Rohde 1992). However, there are several models that predict such a direct relationship from either the turnover of species or the turnover of individuals. First, the classic species-turnover model that underlies the theory of island biogeography suggests that low energy (food) levels lead to low population sizes, and, consequently, higher extinction rates. This could lead to lower species numbers at dynamic equilibrium when energy levels are low (Hutchinson 1959, Turner 1992, Turner et al. 1996). Second, minimum viable population sizes may be lower in areas of high productivity (D. J. Currie et al., unpublished manuscript). This would also lead to greater species richness in warm, wet climates. Third, Hubbell's "neutral theory of biodiversity" (Hubbell 2001) uses a set of null assumptions about the turnover of individuals that predicts that species richness (via the "fundamental biodiversity number") will be directly related to the productivity and area of the metacommunity, as well as the speciation rate. Thus, there are three models which predict that population sizes, or the total number of individuals over all species, will influence the equilibrium number of species, and any environmental factor that influences population sizes across the appropriate spatial scale is therefore capable of influencing or controlling species richness. The productivity hypothesis proposes that the overriding factor is food supply. The ambient energy hypothesis assumes that growth and reproduction are greater at higher temperatures, and winter mortality also will be lower, leading to higher population sizes. This is obvious for plants and ectotherms, but is also true for mammals and birds, because higher overall temperatures allow endotherms to divert metabolic energy that might otherwise be spent on maintaining body temperature into growth and reproduction (Root 1988, Wright et al. 1993, Turner et al. 1996). Clearly, under very hot and arid conditions the availability of water per se will become the limiting factor.

Of course not all climatic variables, even those generally related to productivity, always have positive relationships with species richness over their entire range. Although curvilinear relationships may be found across all spatial scales (Mittelbach et al. 2001), at large scales these are likely to occur because these variables do not in fact correlate with productivity over their entire range (e.g., O'Brien 1993, Scurlock and Olson 2002, Francis and Currie 2003). Alternatively, there are hierarchical controls on richness, illustrated by the relationships between PET and bird/butterfly richness in northern latitudes (see Fig. 2), where beyond a minimum threshold other factors become limiting.

Unquestionably, climate is strongly associated with richness gradients, but how well do climatic variables explain richness? In some cases, a single energy or water variable can by itself account for $>90 \%$ of the variance in species richness (Table 1). But in others, the best predictor accounts for substantially less than half of the variance, raising the possibility that alternative, unmeasured factors may be better predictors. For example, it is widely assumed that the grain size and extent of the analysis has a strong influence on the processes driving richness. At small grain sizes and local extents a wide range of biotic and abiotic factors are important, whereas at large grain sizes and continental/global extents climatic and historical factors dominate (Whittaker et al. 2001, Willis and Whittaker 2002). There are also several examples showing how differences in grain size can influence the contributions of variables to regression models of richness (Rahbek and Graves 2001, Hurlbert and Haskell 2003). However, when we distinguished studies based on sampled data (i.e., richness measured over very local scales) vs. those based on range maps (i.e., richness estimated within large areas), we found no difference in the explanatory power of climate variables. Presumably, the range of climatic variation across the large extents considered here is sufficient to swamp the effects of any additional factors influencing richness, irrespective of the grain size. This issue will be further explored elsewhere. Even so, single climatic variables are sometimes insufficient to describe richness gradients very well, even over large spatial extents. In some of these cases, the inclusion of additional climatic variables substantially improved the predictive power of the models (see original papers listed in Table 1), but in others, most of the variance was not explained by any of the measured variables.

In closing, we note that our evaluation of the energy hypothesis should be viewed with some caveats. First, we do not claim that water-energy dynamics accounts for broad-scale richness variation in all taxonomic groups, nor that it is the sole factor driving richness of any single group. For example, the extent that the evolutionary history of different regions influences the global diversity gradient remains contentious (see e.g., Haydon et al. 1993, Latham and Ricklefs 1993, Schluter and Ricklefs 1993, McGlone 1996, Whittaker and Field 2000, Francis and Currie 2003, Hawkins et al. 2003), and we cannot exclude the possibility that biotic factors affect diversity at large grains and extents (e.g., 
Kaufman 1995). Even so, it is now clear that the link between water-energy and richness is widespread and generally strong, identifying it as a key component of future biodiversity research. Second, our conclusions are necessarily based on correlations rather than experimental evidence, making it difficult to tease apart direct and indirect effects of climate on richness and on underlying mechanisms. Thus, our interpretation must be tentative until mechanisms can be evaluated based on any mutually exclusive predictions each makes. Further, direct comparisons of energy to other hypotheses are challenging, owing to the varying natures of proposed explanations and to the difficulty in evaluating opposing hypotheses within a uniform framework. Finally, we have focused on terrestrial systems, although many of our arguments may also apply to marine and freshwater systems (e.g., Rex et al. 1993, Oberdorff et al. 1995, Roy et al. 1998, Rutherford et al. 1999). It is hoped that our synthesis will stimulate others to test the generality of these ideas in aquatic habitats.

\section{ACKNOWLEDGMENTS}

This work was conducted as part of the Energy and Geographic Variation in Species Richness Working Group supported by the National Center for Ecological Analysis and Synthesis, a Center funded by NSF (Grant No. DEB0072909), the University of California, and the Santa Barbara campus. Additional support was also provided for the Postdoctoral Associate in the Group (D. M. Kaufman), as well as sabbatical support for G. G. Mittelbach.

\section{Literature Cited}

Adams, J. M., and F. I. Woodward. 1989. Patterns in tree species richness as a test of the glacial extinction hypothesis. Nature 339:699-701.

Allen, A. P., J. H. Brown, and J. F. Gillooly. 2002. Global biodiversity, biochemical kinetics, and the energetic-equivalence rule. Science 297:1545-1548.

Andrews, P., and E. M. O’Brien. 2000. Climate, vegetation, and predictable gradients in mammal species richness in southern Africa. Journal of Zoology 251:205-231.

Badgley, C., and D. L. Fox. 2000. Ecological biogeography of North American mammals: species density and ecological structure in relation to environmental gradients. Journal of Biogeography 27:1437-1467.

Balmford, A., J. L. Moore, T. Brooks, N. Burgess, L. A. Hansen, P. Williams, and C. Rahbek. 2001. Conservation conflicts across Africa. Science 291:2616-2619.

Brown, J. H. 1973. Species diversity of seed-eating desert rodents in sand dune habitats. Ecology 54:775-787.

Brown, J. H. 1981. Two decades of homage to Santa Rosalia: toward a general theory of diversity. American Zoologist 21:877-888.

Clinebell, R. R., O. L. Phillips, A. H. Gentry, N. Stark, and H. Zuring. 1995. Prediction of neotropical tree and liana species richness from soil and climatic data. Biodiversity and Conservation 4:56-90.

Colwell, R. K., and D. C. Lees. 2000. The mid-domain effect: geometric constraints on the geography of species richness. Trends in Ecology and Evolution 15:70-76.

Connell, J. H., and E. Orias. 1964. The ecological regulation of species diversity. American Naturalist 98:399-414.

Currie, D. J. 1991. Energy and large-scale patterns of animaland plant-species richness. American Naturalist 137:2749.
Currie, D. J., and V. Paquin. 1987. Large-scale biogeographical patterns of species richness of trees. Nature 329:326327.

Danell, K., P. Lundberg, and P. Niemelä. 1996. Species richness in mammalian herbivores: patterns in the boreal zone. Ecography 19:404-409.

Davidson, D. W. 1977. Species diversity and community organization in desert seed-eating ants. Ecology 58:711-724.

Dingle, H., W. A. Rochester, and M. P. Zalucki. 2000. Relationships among climate, latitude and migration: Australian butterflies are not temperate-zone birds. Oecologia 124:196-207.

Dynesius, M., and R. Jansson. 2000. Evolutionary consequences of changes in species' geographical distributions driven by Milankovitch climate oscillations. Proceedings of the National Academy of Sciences of the United States of America 97:9115-9120.

Eggleton, P., P. H. Williams, and K. J. Gaston. 1994. Explaining global termite diversity: productivity or history? Biodiversity and Conservation 3:318-330.

Francis, A. P., and D. J. Currie. 1998. Global patterns of tree species richness in moist forests: another look. Oikos 81: 598-602.

Francis, A. P., and D. J. Currie. 2003. A globally consistent richness-climate relationship for angiosperms. American Naturalist 161:523-536.

Ganzhorn, J. U., S. Malcomber, O. Andrianantoanina, and S. M. Goodman. 1997. Habitat characteristics and lemur species richness in Madagascar. Biotropica 29:331-343.

Gentry, A. H. 1982. Patterns of neotropical plant-species diversity. Evolutionary Biology 15:1-85.

Gentry, A. H. 1988. Changes in plant community diversity and floristic composition on environmental and geographical gradients. Annals of the Missouri Botanical Garden 75: $1-34$.

Glaser, P. H. 1992. Raised bogs in eastern North America: regional controls for species richness and floristic assemblages. Journal of Ecology 80:535-554.

Goetz, S. J., S. D. Prince, J. Small, A. Gleason, and M. M. Thawley. 2000. Interannual variability in global terrestrial primary production: results of a model driven with satellite observations. Journal of Geophysical Research 105(D15): 20077-20091.

Hawkins, B. A., and J. A. F. Diniz-Filho. 2002. The middomain effect cannot explain the diversity gradient of Nearctic birds. Global Ecology and Biogeography 11:419426.

Hawkins, B. A., E. E. Porter, and J. A. F. Diniz-Filho. 2003. Productivity and history as predictors of the latitudinal diversity gradient for terrestrial birds. Ecology 84:16081623.

Haydon, D., R. R. Radtkey, and E. R. Pianka. 1993. Experimental biogeography: interactions between stochastic, historical, and ecological processes in a model archipelago. Pages 117-130 in R. E. Ricklefs and D. Schluter, editors. Species diversity in ecological communities: historical and geographical perspectives. University of Chicago Press, Chicago, Illinois, USA.

Heatwole, H. 1976. Reptile ecology. University of Queensland Press, St. Lucia, Australia.

Heggberget, T. M. 1987. Number and proportion of southern bird species in Norway in relation to latitude, spring temperature and respiration equivalent. Holarctic Ecology 10: 81-89.

Hubbell, S. P. 2001. The unified neutral theory of biodiversity and biogeography. University of Princeton Press, Princeton, New Jersey, USA.

Hurlbert, A. H., and J. P. Haskell. 2003. The effect of energy and seasonality on avian species richness and community composition. American Naturalist 161:83-97.

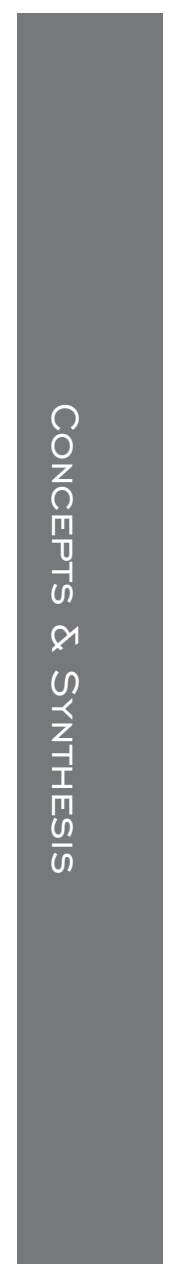


Huston, M. A. 1979. A general hypothesis of species diversity. American Naturalist 113:81-101.

Huston, M. A. 1994. Biological diversity. Cambridge University Press, Cambridge, UK.

Huston, M. A. 1999. Local processes and regional patterns: appropriate scales for understanding variation in the diversity of plants and animals. Oikos 86:393-401.

Hutchinson, G. E. 1959. Homage to Santa Rosalia or why are there so many kinds of animals? American Naturalist 93:145-159.

Kaspari, M., S. O'Donnell, and J. R. Kercher. 2000. Energy, density, and constraints to species richness: ant assemblages along a productivity gradient. American Naturalist 155:280-293.

Kaufman, D. M. 1995. Diversity of New World mammals: universality of the latitudinal gradients of species and bauplans. Journal of Mammalogy 76:322-334.

Kay, R. F., R. H. Madden, C. Van Schaik, and D. Higdon. 1997. Primate species richness is determined by plant productivity: implications for conservation. Proceedings of the National Academy of Sciences of the United States of America 94:13023-13027.

Kerr, J. T., and D. J. Currie. 1999. The relative importance of evolutionary and environmental controls on broad-scale patterns of species richness in North America. Ecoscience 6:329-337.

Kerr, J. T., and L. Packer. 1997. Habitat heterogeneity as a determinant of mammal species richness in high-energy regions. Nature 385:252-254.

Kerr, J. T., and L. Packer. 1999. The environmental basis of North American species richness patterns among Epicauta (Coleoptera: Meloidae). Biodiversity and Conservation 8: 617-628.

Kerr, J. T., T. R. E. Southwood, and J. Cihlar. 2001. Remotely sensed habitat diversity predicts butterfly species richness and community similarity in Canada. Proceedings of the National Academy of Sciences of the United States of America 98:11365-11370.

Kerr, J. T., R. Vincent, and D. J. Currie. 1998. Lepidopteran richness patterns in North America. Ecoscience 5:448-453.

Kessler, M. 2001. Pteridophyte species richness in Andean forests in Bolivia. Biodiversity and Conservation 10:14731495.

Latham, R. E., and R. E. Ricklefs. 1993. Global patterns of tree species richness in moist forests: energy-diversity theory does not account for variation in species richness. Oikos 67:325-333.

Lennon, J. J., J. J. D. Greenwood, and J. R. G. Turner. 2000. Bird diversity and environmental gradients in Britain: a test of the species-energy hypothesis. Journal of Animal Ecology 69:581-598.

Lieth, H. 1975. Modeling the primary productivity of the world. Pages 237-263 in H. Lieth and R. H. Whittaker, editors. Primary productivity of the biosphere. SpringerVerlag, New York, New York, USA.

Lobo, J. M., J. P. Lumaret, and P. Jay-Robert. 2002. Modelling the species richness distribution of French dung beetles (Coleoptera, Scarabaeidae) and delimiting the predictive capacity of different groups of explanatory variables. Global Ecology and Biodiversity 11:265-277.

McGlone, M. S. 1996. When history matters: scale, time, climate and tree diversity. Global Ecology and Biogeography Letters 5:309-314.

Medellin, R. A. 1994. Mammal diversity and conservation in the Selva-Lacandona, Chiapas, Mexico. Conservation Biology 8:780-799.

Meserve, P. L., and W. E. Glanz. 1978. Geographical ecology of small mammals in the northern Chilean arid zone. Journal of Biogeography 5:135-148.
Mittelbach, G. G., C. F. Steiner, S. M. Scheiner, K. L. Gross, H. L. Reynolds, R. B. Waide, M. R. Willig, S. I. Dodson, and L. Gough. 2001. What is the observed relationship between species richness and productivity? Ecology 82: 2381-2396.

Mourelle, C., and E. Ezcurra. 1996. Species richness of Argentine cacti: a test of biogeographic hypotheses. Journal of Vegetation Science 7:667-680.

Oberdorff, T., J. F. Guégan, and B. Hugueny. 1995. Global scale patterns in freshwater fish species diversity. Ecography $\mathbf{1 8}: 345-352$.

O’Brien, E. M. 1993. Climatic gradients in woody plantspecies richness: towards an explanation based on an analysis of southern Africa woody flora. Journal of Biogeography 20:181-198.

O’Brien, E. M. 1998. Water-energy dynamics, climate, and prediction of woody plant species richness: an interim general model. Journal of Biogeography 25:379-398.

Pearson, D. L., and S. S. Carroll. 1998. Global patterns of species richness: spatial models for conservation planning using bioindicator and precipitation data. Conservation $\mathrm{Bi}-$ ology 12:809-821.

Pianka, E. R. 1966. Latitudinal gradients in species diversity: a review of concepts. American Naturalist 100:33-46.

Pianka, E. R. 1967. On lizard species diversity: North American flatland deserts. Ecology 48:333-351.

Pianka, E. R., and J. J. Schall. 1981. Species densities of Australian vertebrates. Pages 1676-1694 in A. Keast, editor. Ecological biogeography of Australia. Dr. W. Junk Publishers, The Hague, The Netherlands.

Rabinovich, J. E., and E. H. Rapoport. 1975. Geographical variation of diversity in Argentine passerine birds. Journal of Biogeography 2:141-157.

Rahbek, C., and G. R. Graves. 2001. Multiple scale assessment of patterns of avian species richness. Proceedings of the National Academy of Sciences of the United States of America 98:4534-4539.

Reed, K. E., and J. G. Fleagle. 1995. Geographic and climatic control of primate diversity. Proceedings of the National Academy of Sciences of the United States of America 92: 7874-7876.

Rex, M. A., C. T. Stuart, R. R. Hessier, J. A. Allen, H. L. Sanders, and G. D. F. Wilson. 1993. Global-scale latitudinal patterns of species diversity in the deep-sea benthos. Nature 365:636-639.

Richerson, P. J., and K. Lum. 1980. Patterns of plant species diversity in California: relation to weather and topography. American Naturalist 116:504-536.

Ritchie, M. E., and H. Olff. 1999. Spatial scaling laws yield a synthetic theory of biodiversity. Nature 400:557-560.

Rohde, K. 1992. Latitudinal gradients in species diversity: the search for the primary cause. Oikos 65:514-527.

Root, T. 1988. Energy constraints on avian distributions and abundances. Ecology 69:330-339.

Rosenzweig, M. L. 1968. Net primary productivity of terrestrial environments: predictions from climatological data. American Naturalist 102:67-84.

Rosenzweig, M. L. 1995. Species diversity in space and time. Cambridge University Press, Cambridge, UK.

Roy, K., D. Jablonski, J. W. Valentine, and G. Rosenberg. 1998. Marine latitudinal diversity gradients: tests of causal hypotheses. Proceedings of the National Academy of Science of the United States of America 95:3600-3702.

Rutherford, S., S. D'Hondt, and W. Prell. 1999. Environmental controls on the geographic distribution of zooplankton diversity. Nature 400:749-753.

SAS Institute. 2000. JMP. Version 4.0. SAS Institute, Cary, North Carolina, USA.

Schall, J. J., and E. R. Pianka. 1977. Species densities of reptiles and amphibians on the Iberian Peninsula. Doñana, Acta Vertebrata 4:27-34. 
Schall, J. J., and E. R. Pianka. 1978. Geographical trends in numbers of species. Science 201:679-686.

Scheibe, J. S. 1987. Climate, competition, and the structure of temperate zone lizard communities. Ecology 68:14241436.

Scheiner, S. M., and J. M. Rey-Benayas. 1994. Global patterns of plant diversity. Evolutionary Ecology 8:331-347.

Schluter, D., and R. E. Ricklefs. 1993. Species diversity: an introduction to the problem. Pages 1-10 in R. E. Ricklefs and D. Schluter, editors. Species diversity in ecological communities: historical and geographical perspectives. University of Chicago Press, Chicago, Illinois, USA.

Schulze, E.-D., R. Ellis, W. Schulze, P. Trimborn, and H. Ziegler. 1996. Diversity, metabolic types and delta C-13 carbon isotope ratios in the grass flora of Namibia in relation to growth form, precipitation and habitat conditions Oecologia 106:352-369.

Scurlock, J. M. O., and R. J. Olson. 2002. Terrestrial net primary productivity: a brief history and a new worldwide database. Environmental Reviews 10:91-109.

Specht, A., and R. L. Specht. 1994. Biodiversity of overstory trees in relation to canopy production and stand density in the climatic gradient from warm temperature to tropical Australia. Biodiversity Letters 2:39-45.

Stephenson, N. L. 1990. Climatic control of vegetation distribution: the role of water balance. American Naturalist 135:649-670.

Terent'ev, P. W. 1963. Attempt at application of analysis of variation to the qualitative richness of the fauna of terrestrial vertebrates of the U. S. S. R. Vestnik Leningradskovo Universiteta 21:19-26. Translated by E. J. Maly and edited by E. R. Pianka, Smithsonian Herpetological Information Services, 1968.

Turner, J. R. G. 1992. Stochastic processes in populations: the horse behind the cart? Pages 29-53 in J. R. Berry, T.
J. Crawford, and G. M. Hewitt, editors. Genes in ecology. Blackwell Scientific, Oxford, UK.

Turner, J. R. G., C. M. Gatehouse, and C. A. Corey. 1987. Does solar energy control organic diversity? Butterflies, moths and the British climate. Oikos 48:195-205.

Turner, J. R. G., J. Lennon, and J. D. Greenwood. 1996. Does climate cause the global biodiversity gradient? Pages 199220 in M. E. Hochberg, J. Clobert, and R. Barbault, editors. Aspects of the genesis and maintenance of biological diversity. Oxford University Press, Oxford, UK.

van Rensburg, B. J., S. L. Chown, and K. J. Gaston. 2002. Species richness, environmental correlates, and spatial scale: a test using South African birds. American Naturalist 159:566-577.

von Humboldt, A. 1808. Ansichten der Natur mit wissenschaftlichen Erlauterungen. J. G. Cotta, Tübingen, Germany.

Whittaker, R. J., and R. Field. 2000. Tree species richness modeling: an approach of global applicability? Oikos 89: 399-402.

Whittaker, R. J., K. J. Willis, and R. Field. 2001. Scale and species richness: towards a general, hierarchical theory of species diversity. Journal of Biogeography 28:453-470.

Willig, M. R., D. M. Kaufman, and R. D. Stevens. 2003. Latitudinal gradients of biodiversity: pattern, process, scale, and synthesis. Annual Review of Ecology, Evolution, and Systematics 34:273-309.

Willis, K. J., and R. J. Whittaker. 2002. Species diversity: scale matters. Science 295:1245-1248.

Wright, D. H. 1983. Species-energy theory: an extension of species-area theory. Oikos 41:496-506.

Wright, D. H., D. J. Currie, and B. A. Maurer. 1993. Energy supply and patterns of species richness on local and regional scales. Pages 66-74 in R. E. Ricklefs and D. Schluter, editors. Species diversity in ecological communities: historical and geographical perspectives. University of Chicago Press, Chicago, Illinois, USA.

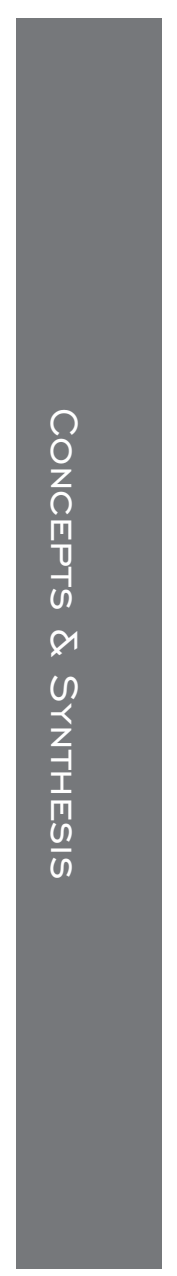

